\title{
A New GFSK Demodulation Technique using EKF in Bluetooth Receivers
}

\author{
Mr. Alhaj-Saleh Abdallah ${ }^{1}$, Dr. Ahmad Nsour ${ }^{2}$, Prof. Mohamed Zohdy ${ }^{3}$ \\ Dept. of Electrical and Computer Engineering, Oakland University, Rochester MI, USA ${ }^{1,2,3}$
}

\begin{abstract}
Bluetooth is a wireless technology that is widely used in consumer electronics and deployed in many industries due to its ease of use and low cost. Gaussian frequency shift keying (GFSK) is the modulation scheme used in Bluetooth receivers to achieve a basic data rate of $1 \mathrm{Mbps}$. In this article, a new non- coherent demodulation technique based on the Extended Kalman Filter (EKF) is presented to detect a GFSK modulated signal in Bluetooth receivers for AWGN channels. The bit error rate (BER), and Packet Error Rate (PER) were used as the performance metrics of the proposed receiver at both the physical and system levels. Simulation results show an improvement in the performance compared to other existing techniques presented in the literature.
\end{abstract}

Keywords: AWGN, Bluetooth, Extended Kalman Filter, Gaussian Frequency Shift Keying, Continues Phase Modulation.

\section{INTRODUCTION}

BLUETOOTH [1] operates in the $2.40 \mathrm{GHz}$ ISM (Industrial, Scientific and Medical) unlicensed band. It uses the Gaussian frequency shift keying (GFSK) modulation scheme, which is a special case of continuous phase modulation (CPM), to achieve a basic data rate of 1 Mbps. Several Bluetooth receivers presented in the literature achieve higher power efficiency at the expense of increased complexity of the receiver design. The power efficiency is the achievable bit error rate (BER) for a certain signal-to-noise power ratio (SNR). The limiter discriminator with an integrator (LDI) receiver [2], phase differential receiver [3], and Bluetooth zero-crossing matched filter (BT-ZXMF) receiver [4] are low-cost and simple to implement but suffer from low power efficiency. A low-complexity simplified maximum a posteriori (MAP) receiver using coherent sequence detection was presented in [5]. It exhibited a $6 \mathrm{~dB}$ improvement over the LDI but relied on a known exact modulation index. Other Bluetooth receivers deployed the Viterbi algorithm to search for the minimum Euclidean distance path through the state trellis [6] [7]; such receivers exhibited a high power efficiency but experience high computational complexity. These receivers also assume a nominal value of the modulation index. For Bluetooth, the modulation index varies from $0: 28$ to $0: 35$. The variations in the modulation index result in varying trellis structures for sequence detection.

A more complex receiver that uses a four-state trellis proposed in the literature is the so called max-logmaximum likelihood LDI (MLM-LDI) receiver [8], which exhibited a $4 \mathrm{~dB}$ improvement over the LDI receiver. In [9], Lampe et al. proposed a high complexity receiver that consisted of a single filter and a subsequent non-coherent sequence detector (NSD) that operates on a two-state trellis, achieving an improvement of more than $4 \mathrm{~dB}$ compared with the LDI. In [10], Ibrahim et al. presented a new receiver design based on Laurent's decomposition. Although the receiver performance was comparable to the maximum likelihood sequence detection (MLSD), the modulation index had to be known; otherwise, the receiver must search for the modulation index through an adaptation period, which causes severe degradation of the performance. In [11], Yu et al. proposed a differential GFSK demodulator that averages the phase based on the SNR maximizing criterion; this receiver had an improvement of 1-2 dB over the LDI receiver.

In this article, a new GFSK demodulation technique is being proposed. The EKF is used for phase estimation, and a low cost detection algorithm is used to make decisions regarding the bit received. The proposed receiver does not require prior knowledge of the modulation index or a predetection filter to reject out-of-band interference. The proposed receiver outperforms other techniques with similar complexity, such as the LDI.

The remainder of the paper is organized as follows. In Section II, introduction of the system definition will be presented. Section III will briefly introduce the Bluetooth transmission channel. In Section IV, a receiver based on EKF will be proposed. Simulation results and discussion will be presented in Section V, followed by the conclusions in Section VI.

\section{GFSK SIGNAL STRUCTURE}

A passband transmitted GFSK signal can be expressed as [3]

$$
s(t, I, h)=\sqrt{\frac{2 E_{s}}{T}} \cos \left\{2 \pi f_{0} t+\varphi(t, I, h)+\varphi_{0}\right\} .
$$

where $E_{S}$ is the energy symbol and $T$ is the symbol duration, $f_{0}$ is the carrier frequency, $\varphi_{0}$ is an arbitrary constant phase shift, and $\varphi(t, I, h)$ is the continuous phase of the signal and can be expressed as

$$
\varphi(t, I, h)=2 \pi h \sum_{k=n-L+1}^{n} q(t-k T) I_{k}+\pi h \sum_{k=-\infty}^{n-L} I_{k} .
$$

where $h$ is the modulation index, $n T \leq t \leq(n+1) \mathrm{T}$ is the total number of transmitted bits, and $\mathrm{I}_{\mathrm{k}} \in\{ \pm 1\}$ represents the binary data. The normalized phase pulse 
$q(t)=\int_{-\infty}^{t} g(\tau) d \tau$ is obtained from the frequency impulse $\mathrm{g}(\mathrm{t})[11]$

$$
\mathrm{g}(\mathrm{t})=\frac{1}{2 \mathrm{~T}}\left(\mathrm{Q}\left(\lambda \cdot \mathrm{BT}\left(\mathrm{t}-\frac{\mathrm{T}}{2}\right)\right)-\mathrm{Q}\left(\lambda \cdot \mathrm{BT}\left(\mathrm{t}+\frac{\mathrm{T}}{2}\right)\right)\right) .
$$

BT is the time bandwidth product of the pre-modulation filter that corresponds to a minimum carrier separation to ensure orthogonality between signals in adjacent channels, $\lambda=2 \pi / \sqrt{\log (2)}$; and $Q(\cdot)$ is the Gaussian Q-function [9]. In Bluetooth standard, $\mathrm{BT}=0.5$, the modulation index varies in the range $0.28<\mathrm{h}<0.35$, and $\mathrm{T}$ is equal to $10^{-6}$ [9].

\section{BLUETOOTH TRANSMISSION CHANNEL}

An AWGN channel is a transmission channel that adds white Gaussian noise to a signal. The ratio between the signal's power (i.e. $\left.\mathrm{P}_{\text {signal }}\right)$ and the noise's power (i.e. $\left.\mathrm{P}_{\text {noise }}\right)$ is expressed by [3]

$$
\mathrm{SNR}_{\mathrm{dB}}=10 \log \frac{\mathrm{P}_{\text {signal }}}{\mathrm{P}_{\text {noise }}} .
$$

This type of channel has been widely considered for Bluetooth performance for several reasons that were described in [12], and they are listed again for completeness. (1) Because Bluetooth communication is deployed mainly in an indoor environment, fast fading does not have significant impact on Bluetooth performance and it can be neglected. (2) Line of sight communication from the receiver to the transmitter exists due to Bluetooth transmission power restrictions. (3) Interfering signals that might affect the performance of Bluetooth, such as IEEE $802.11 \mathrm{~b}$ operating in the same ISM band, can be approximated by noise with a constant spectral density.

\section{PROPOSED RECEIVER}

\section{A. GFSK received signal}

The complex envelope of the received signal may be expressed as [9]

$$
r(t)=s(t, I, h) e^{j \omega(t)}+\gamma(t) .
$$

where the phase rotation $\omega(t)$ is modeled as a random variable with uniform distribution in interval $[0,2 \pi)$, and it is assumed to be slowly varying such that it can be considered constant over the symbol period. $\gamma(t)$ is a complex-valued Gaussian white noise processes with independent components each with two sided power density $N_{0}$ [9] . Sampling the received signal from (Eq. 3) at time $k T, r_{k}$ can be expressed as [11]

$$
r(k)=s\left(k, I_{k}, h_{k}\right) e^{j \omega_{k}}+\gamma_{k} .
$$

Extracting the sampled amplitude $A_{k}$ and phase $\theta_{k}$ from the received signal $r(k)$ yields,

$$
\begin{gathered}
A_{k}=|r(k)| . \\
\theta_{k}=\arg \{r(k)\} .
\end{gathered}
$$

It is worth noting that the amplitude of the continuous signal is assumed to be constant over a packet length, but it may change between packets [9]. Following the derivation in [14], $\theta_{k}$ may be expressed as

$$
\theta_{k}=\omega_{k}+\phi_{k}+\psi_{k} \text {. }
$$

where $\psi_{k}=\arg \left[\gamma_{k}\right]=\gamma_{k-1}+\varepsilon_{k}$ represent the phase of sampled complex-valued Gaussian white noise process $\gamma_{t}$, and $\left\{\varepsilon_{k}\right\}$ are real, independent, and identically distributed Gaussian random variables, with $N\left(0, \sigma_{\varepsilon}\right)$ [14] . $\phi_{k}$ signify the transmitted signal phase and can be represented by

$$
\phi_{k}=\arg \left[s\left(k, I_{k}, h_{k}\right)\right]=\phi_{k-1}+\pi I_{k}\left(h_{r x}+h_{e}\right) \text {. }
$$

where $h_{r x}$ is the assumed receiver modulation index (i.e. $h_{r x}=r / p$ where $r$ and $p$ are relatively prime integers), and $h_{e}$ is the modulation index mismatch between the transmitter and receiver (i.e. $h_{e}=h_{r x}-h_{t x}$ ), and it is assumed to be unknown and modeled as a random variable with known distribution [14]. $\quad \omega_{k}=\omega(k T)$ indicates phase rotation and it can be represented as [11]

$$
\omega_{k}=\omega_{k-1}+\xi_{k} .
$$

where $\left\{\xi_{k}\right\}$ are real, independent, and identically distributed Gaussian random variables, with $N\left(0, \sigma_{\xi}\right)$ [14]. Using the above equations, $\theta_{k}$ may be expressed as [14]

$\theta_{k}=\omega_{k-1}+\xi_{k}+\phi_{k-1}+\pi I_{k}\left(h_{r x}+h_{e}\right)+\gamma_{k-1}+\varepsilon_{k} \cdot(10)$

B. GFSK state and measurement equations

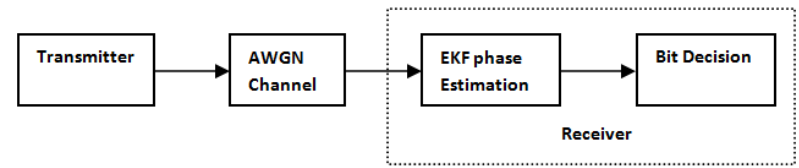

Fig.1. Receiver architecture

The proposed receiver architecture is shown in Fig. 1. A state space transition model is derived to non-coherently estimate the phase of the GFSK modulated signal. Let $\theta$ and $r$ denote the state and measurement representation of the state space model respectively. Assuming that the state $\theta_{k}$ is equal to the sampled received phase $\theta(k T)$, and there is no external effect to the system (i.e. $u(\cdot)=0$ ), the EKF transition and observation model may be expressed as [14]

$$
\begin{aligned}
& \theta_{\mathrm{k}}=\mathrm{f}\left(\theta_{\mathrm{k}-1}, 0\right)+\mathrm{w}_{\mathrm{k}-1} . \\
& \mathrm{r}_{\mathrm{k}}=\mathrm{h}\left(\theta_{\mathrm{k}}\right)+\mathrm{v}_{\mathrm{k}} .
\end{aligned}
$$

where

$$
\begin{aligned}
& \mathrm{f}\left(\theta_{\mathrm{k}-1}\right)=\theta_{\mathrm{k}-1}+\pi \mathrm{I}_{\mathrm{k}}\left(\mathrm{h}_{\mathrm{rx}}+\mathrm{h}_{\mathrm{e}}\right)+\xi_{\mathrm{k}}+\varepsilon_{\mathrm{k}} . \\
& \mathrm{h}\left(\theta_{\mathrm{k}}\right)=\mathrm{A}_{\mathrm{k}} \exp \left\{\mathrm{j} \theta_{\mathrm{k}}\right\} .
\end{aligned}
$$

and $\mathrm{w}_{\mathrm{k}-1}$ and $\mathrm{v}_{\mathrm{k}}$ are independent and identically distributed i.i.d. process and measurement Gaussian noises with zero mean and covariance's $Q_{k-1}$ and $R_{k}$, respectively[15] [16].

\section{EKF algorithm}

Let us assume that $\hat{\theta}_{0}^{+}$and $\mathrm{P}_{0}^{+}$are the initial state and covariance of the EKF, respectively, the time update and measurement update algorithm are described in Table I [3, Ch. 13].

The state transition $\mathrm{F}_{\mathrm{k}-1}$ and the observation $\mathrm{H}_{\mathrm{k}}$ are defined by obtaining the derivatives of equations (8) and (9) with respect to the state

$$
\mathrm{F}_{\mathrm{k}-1}=\left.\frac{\partial \mathrm{f}}{\partial \theta}\right|_{\widehat{\theta}_{\mathrm{k}-1 \mid \mathrm{k}-1}} .
$$




\section{TABLE I EKF Algorithm}

$$
\begin{aligned}
& \text { Time Update (Prediction Step) } \\
& \text { (1) Project the state ahead } \\
& \hat{\theta}_{k \mid k-1}=f\left(\hat{\theta}_{k-1 \mid k-1}\right) \\
& \text { (2) Project the covariance ahead } \\
& \quad P_{k \mid k-1}=F_{k-1} P_{k-1 \mid k-1} F_{k-1}^{T}+Q_{k-1} \\
& P_{k \mid k-1} \text { and } \hat{\theta}_{k \mid k-1} \text { are the a priori covariance and the a priori } \\
& \text { estimate, respectively. } \\
& \text { Measurement Update (Correction Step) } \\
& \text { (1) Compute the Kalman gain } \\
& \quad K_{k}=P_{k \mid k-1} H_{k}^{T}\left(H_{k} P_{k \mid k-1} H_{k}^{T}+R_{k}\right)^{-1} \\
& \text { (2) Update estimate with measurement } r_{k} \\
& \hat{\theta}_{k \mid k}=\hat{\theta}_{k \mid k-1}+K_{k}\left(y_{k}-h\left(\hat{\theta}_{k \mid k-1}\right)\right) \\
& \text { (3) Update the error covariance } \\
& P_{k \mid k}=\left(I-K_{k} H_{k}\right) P_{k \mid k-1} \\
& \hat{\theta}_{k \mid k} \text { and } P_{k \mid k} \text { are the a posteriori estimate and a posteriori } \\
& \text { covariance. }
\end{aligned}
$$

\section{Measurement Update (Correction Step)}

$$
\mathrm{H}_{\mathrm{k}}=\left.\frac{\partial \mathrm{h}}{\partial \theta}\right|_{\widehat{\theta}_{\mathrm{k} \mid \mathrm{k}-1}}=\mathrm{A}_{\mathrm{k}} \mathrm{j} \exp \left\{\mathrm{j} \theta_{\mathrm{k}}\right\} .
$$

\section{Decision Making}

Similar to the conventional differential demodulator technique [3], Ch. 7], the decision algorithm depends on obtaining the instantaneous change $\Delta \widehat{\theta}_{\mathrm{k}}$ from the estimated states by calculating the difference between the current $\hat{\theta}_{\mathrm{k}}$ and the neighboring state $\hat{\theta}_{\mathrm{k}-1}$ [3]

$$
\Delta \hat{\theta}_{\mathrm{k}}=\hat{\theta}_{\mathrm{k}}-\hat{\theta}_{\mathrm{k}-1} \text {. }
$$

The decision then can be made on the sign of $\Delta \widehat{\theta}_{\mathrm{k}}$ (i.e. "1" if the sign is positive, "0" otherwise) [ [3], Ch. 7]. However, due to the phase wrapping issue, the estimated states $\hat{\theta}_{\mathrm{k}}$ are unwrapped by simply adding $\pm 2 \pi$ when the absolute change between the consecutive phase samples are greater than the jump tolerance $\pi[3]$.

\section{PERFORMANCE RESULTS AND DISCUSSION}

In this section, the performance of the proposed receiver is evaluated with extensive MATLAB simulations. Fig. 2 shows the comparison between the transmitted phases, the unfiltered received phases, and the estimated phases using EKF, (i.e. $\operatorname{hrx}=1 / 3, \mathrm{SNR}=30$ ). The results show the EKF data are tracking the transmitted phases very well.

In addition, the performance of the proposed receiver is studied and analyzed at the physical level layer in term of bit error rate (BER).

Simulation of the commonly used LDI receiver [2] as well as the optimized differential receiver [11] were performed and compared to the proposed receiver since all have a similar level of complexity. The complexity of EKF can be easily derived from Table I and it is $\mathrm{O}(\mathrm{N})$ [13]. Also, comparison to the GFSK theoretical performance limit, which is the coherent maximum likelihood sequence detection (MLSD), was performed [3], Ch. 3].

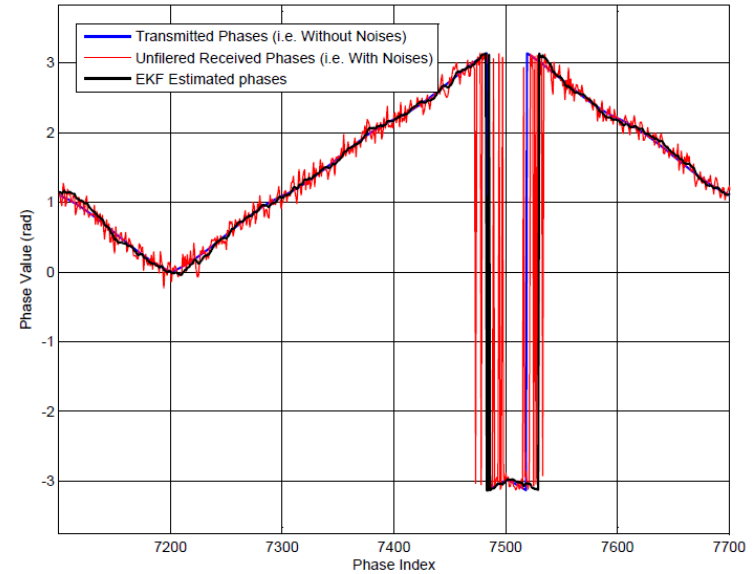

Fig. 2. Transmitted phases vs. Phase estimation using EKF for $\mathrm{SNR}=30$.

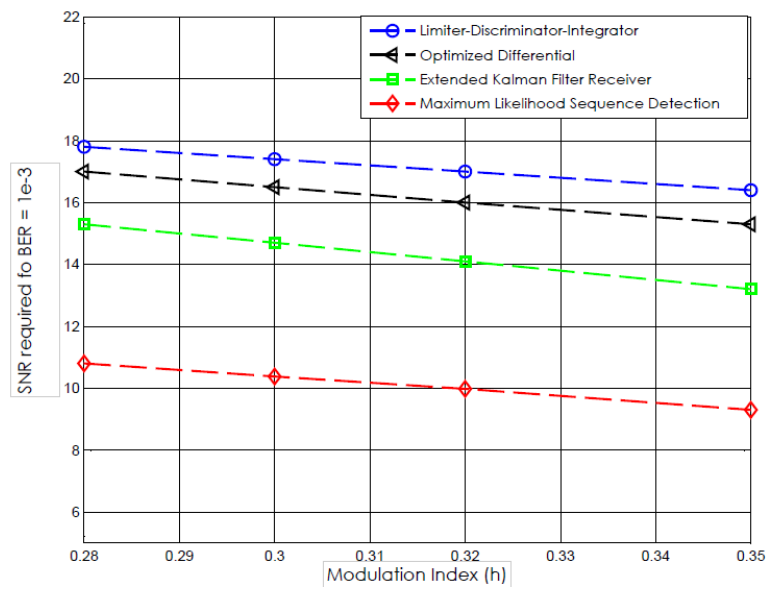

Fig. 3. Physical level performance of the proposed EKF detector as a function of the modulation index $(\mathrm{h})$.

Fig. 3 shows the performance of the proposed receiver in terms of the SNR for different modulation index values (i.e. $0.28<\square<0.35$ ) that will achieve a BER = $10^{-3}$, which is the required BER in the Bluetooth specification [1]. The proposed receiver shows an improvement of approximately $3-4 \mathrm{~dB}$ for different modulation index values compared with the LDI receiver. Furthermore, the system level performance was studied in terms of packet error rate (PER). PER measures the number of packets that are dropped after error correction is applied. Bluetooth uses Synchronous Connection Oriented (SCO) links for audio and they work at $64 \mathrm{kbps}$. SCO link uses three different types of voice packets HV1, HV2 and HV3 providing 10, 20 and 30 bytes of data respectively. The length of voice packets is 366 bits as shown in Fig. 4.

\begin{tabular}{|c|c|c|}
\hline 72 bits & 54 bits & 240 bits \\
\hline Access Code & Header & Payload \\
\hline
\end{tabular}

\section{Fig. 4. SCO packet structure}

A repetition code of rate $1 / 3$ is applied to the header; errors in the access code or the header will lead to a packet drop, a block code with minimum distance equal to 14 so that up to 13 errors can be detected and 6 can be corrected. Table II describes action taken when errors occur in the access code, the header and the payload for the different types of voice packets [1]. 
TABLE II Action Taken When Errors Occur After Correction for Different Voice Packagets

\begin{tabular}{|c|c|c|}
\hline $\begin{array}{c}\text { Error } \\
\text { Location }\end{array}$ & $\begin{array}{c}\text { Error } \\
\text { Correction }\end{array}$ & Action Taken \\
\hline Access code & dmin $=14$ & Packet dropped \\
\hline Packet Header & $1 / 3$ repetition & Packet dropped \\
\hline HV1 payload & $1 / 3$ repetition & Packet accepted \\
\hline HV2 payload & $2 / 3$ block code & Packet accepted \\
\hline HV3 payload & No FEC & Packet accepted \\
\hline
\end{tabular}

In all simulations, two types of Bluetooth voice packets (i.e., HV1 and HV3) were used in which 100 samples per symbol and $1 \mathrm{~mW}$ of transmission power were set. The distance between the Bluetooth transmitter and receiver is $5 \mathrm{~m}$ [17]. Fig. 5 shows the performance of the proposed receiver in terms of PER for two voice packets (i.e., HV1 and HV3) for a modulation index of $1 / 3$. The proposed receiver shows PER improvement over LDI and the differential detector.

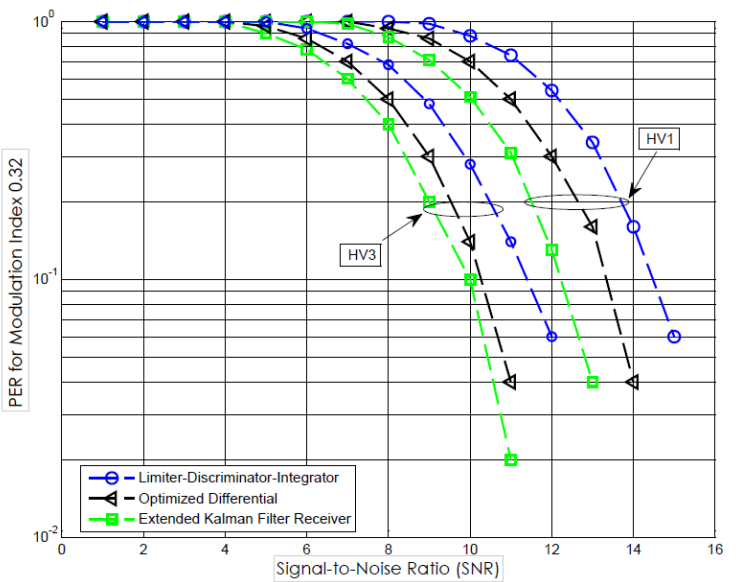

Fig.5. System layer performance of the proposed EKF detector. PER vs.SNR for modulation indexes $(h)=1 / 3$.

Finally, the run time of computational complexities and performance of EKF are investigated on a computer with an Intel Core i7 with a CPU of $2.8 \mathrm{GHz}$ and $8 \mathrm{~Gb}$ RAM, running Windows 7 service Pack 1 . The run time computational complexity study is conducted using MATLAB software Profiler functionality which is used to debug and optimize MATLAB code files by tracking their execution time. Thus, the computational complexity study is conducted by measuring the total time required to perform one step of the iteration.

Table III Execution Time of Filtering A Phase Using EKF Filter.

\begin{tabular}{|c|c|}
\hline \multicolumn{2}{|c|}{ Run Time Complexity Calculation of EKF Filter } \\
\hline Number of Steps & Total Execution Time (s) \\
\hline 1 & $2.6420 \mathrm{e}-7$ \\
\hline
\end{tabular}

\section{CONCLUSION}

In this paper, a new GFSK demodulator for Bluetooth receivers is proposed based on Kalman filtering theory. EKF was used to estimate the transmitted signal phases, and a low complex detection algorithm was designed to make a decision on the estimated sample. The proposed receiver does not require a prior knowledge of the modulation index or a pre-detection filter for detecting GFSK modulated signals. The performance of this demodulator has been studied by extensive MATLAB simulations at both the system and the physical layers of the design. It was shown that the EKF estimated phases track the transmitted phases with good accuracy, as well as it outperforms the LDI receiver and the optimized differential receiver in terms of BER and PER. The proposed receiver outperforms the LDI receiver by approximately $4 \mathrm{~dB}$ for the AWGN channel noise for the different modulation index values allowed in Bluetooth and as close as $3 \mathrm{~dB}$ to the MLSD bound.

\section{REFERENCES}

[1] Bluetooth SIG, "Specification of the Bluetooth System," Core Version 4.0, 30 June 2010 .

[2] A. Soltanian and R. E. Van Dyck, "Physical layer performance for coexistence of Bluetooth and IEEE $802.11 \mathrm{~b}$ ", in Virginia Tech Symposium on Wireless Personal Communications, June 2001.

[3] J. B. Anderson, T. Aulin and C. E. Sundberg. Digital Phase Modulation. New York, Plenum Press, 1986

[4] Scholand, T.; Jung, P., "Novel receiver structure for Bluetooth based on modified zero-crossing demodulation," Global Telecommunications Conference, 2003. GLOBECOM '03. IEEE, vol.2, no., pp.729,733 Vol.2, 1-5 Dec. 2003

[5] Schiphorst, R.; Hoeksema, R.W.; Slump, C.H., "A (simplified) Bluetooth maximum a posteriori probability (MAP) receiver," Signal Processing Advances in Wireless Communications, 2003. SPAWC 2003. 4th IEEE Workshop on , vol., no., pp.160,164, 15-18 June 2003.

[6] Soltanian, A.; Van Dyck, R.E., "Performance of the Bluetooth system in fading dispersive channels and interference," Global Telecommunications Conference, 2001. GLOBECOM '01. IEEE, vol.6, no., pp.3499,3503 vol.6, 2001.

[7] Wang Jia; Nallanathan, A.; Garg, H.K. "Performance of a Bluetooth system in multipath fading channels and interference," Mobile and Wireless Communications Network, 2002. 4th International Workshop on , vol., no., pp.579,582, 2002.

[8] Scholand, T.; Waadt, A.; Jung, P., "Max-log-ML symbol estimation postprocessor for intermediate frequency LDI detectors," Electronics Letters, vol.40, no.3, pp.183,185, 5 Feb. 2004.

[9] L. Lampe and R. Schober; "Noncoherent Sequence Detection Receiver for Bluetooth Systems" , IEEE Journal on Selected Areas in Communications, Vol.23, no.9, pp. 1718-1727, Sep. 2005.

[10] Ibrahim, N..; Lampe, L.; Schober, R., "Bluetooth Receiver Design Based on Laurent's Decomposition," Vehicular Technology, IEEE Transactions on , vol.56, no.4, pp.1856,1862, July 2007.

[11] Bo Yu; Liuqing Yang; Chia-Chin Chong, "Optimized Differential GFSK Demodulator," Communications, IEEE Transactions on , vol.59, no.6, pp.1497,1501, June 2011.

[12] J. R. Luque, M. J. Moron and E. Casilari. "Analytical and empirical evaluation of the impact of Gaussian noise on the modulations employed by Bluetooth Enhanced Data Rates." EURASIP Journal on Wireless Communications and Networking, Vol.2, pp 1-11, March 2012

[13] D. Simon. Optimal State Estimation: Kalman, H [Infinity] and Nonlinear Approaches. New Jersey: John Wiley and Sons, 2006.

[14] M. Messai, G. Colavolpe, K. Amis, and F. Guilloud, "Robust detection of binary cpms with unknown modulation index," Communications Letters, IEEE, vol. 19, no. 3, pp. 339-342, March 2015.

[15] A. Nsour, A.-S. Abdallah, and M. Zohdy, "Phase tracking in Bluetooth receivers using extended Kalman filtering," in Wireless Communications, Vehicular Technology, Information Theory and Aerospace Electronic Systems (VITAE), 2013 3rd International Conference on, June 2013, pp. 1-5.

[16] A. Nsour, A.-S. Abdallah, and M. Zohdy, "An investigation into using Kalman filtering for phase estimation in Bluetooth receivers for Gaussian and non-Gaussian noise," in Electro/ Information Technology (EIT), 2013 IEEE International Conference on, May 2013, pp. 1-5.

[17] A. Nsour, A.-S. Abdallah, and M. Zohdy, "Non-coherent detection of GFSK using extended Kalman filtering for non-Gaussian noise," in Wireless Telecommunications Symposium (WTS), 2014, April 2014, pp. 1-6.

[18] A. Nsour, A.-S. Abdallah, and M. Zohdy, "GFSK phase estimation using extended Kalman filtering for non-Gaussian noise," in Wireless Telecommunications. 